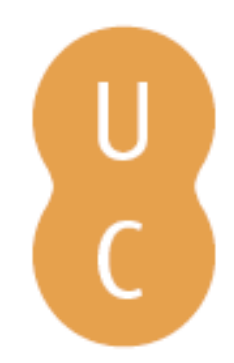

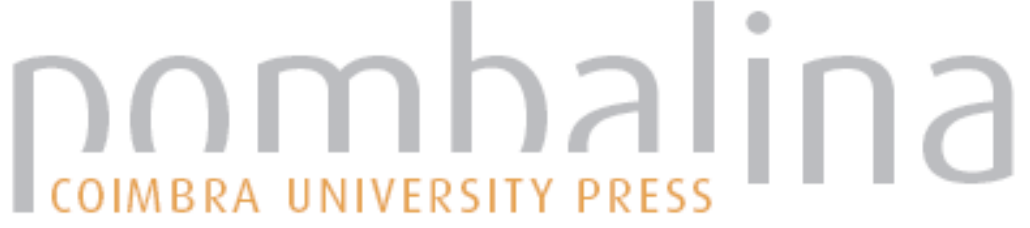

\section{La gestión de la calidad en el curriculum de los títulosde grado y máster en biblioteconomía y documentación}
Autor(es):
Arias Coello, Alicia; Simón Martín, José
Publicado por: Imprensa da Universidade de Coimbra
URL
persistente:
URI:http://hdl.handle.net/10316.2/31904
DOI:
DOI:http://dx.doi.org/10.14195/978-989-26-0319-3_23
Accessed : $\quad$ 26-Apr-2023 12:56:41

A navegação consulta e descarregamento dos títulos inseridos nas Bibliotecas Digitais UC Digitalis, UC Pombalina e UC Impactum, pressupõem a aceitação plena e sem reservas dos Termos e Condições de Uso destas Bibliotecas Digitais, disponíveis em https://digitalis.uc.pt/pt-pt/termos.

Conforme exposto nos referidos Termos e Condições de Uso, o descarregamento de títulos de acesso restrito requer uma licença válida de autorização devendo o utilizador aceder ao(s) documento(s) a partir de um endereço de IP da instituição detentora da supramencionada licença.

Ao utilizador é apenas permitido o descarregamento para uso pessoal, pelo que o emprego do(s) título(s) descarregado(s) para outro fim, designadamente comercial, carece de autorização do respetivo autor ou editor da obra.

Na medida em que todas as obras da UC Digitalis se encontram protegidas pelo Código do Direito de Autor e Direitos Conexos e demais legislação aplicável, toda a cópia, parcial ou total, deste documento, nos casos em que é legalmente admitida, deverá conter ou fazer-se acompanhar por este aviso.

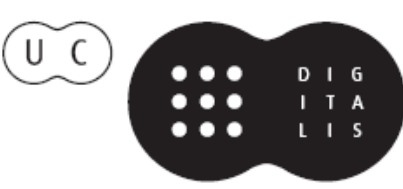


Maria Manuel Borges

Elias Sanz Casado

Coordenação

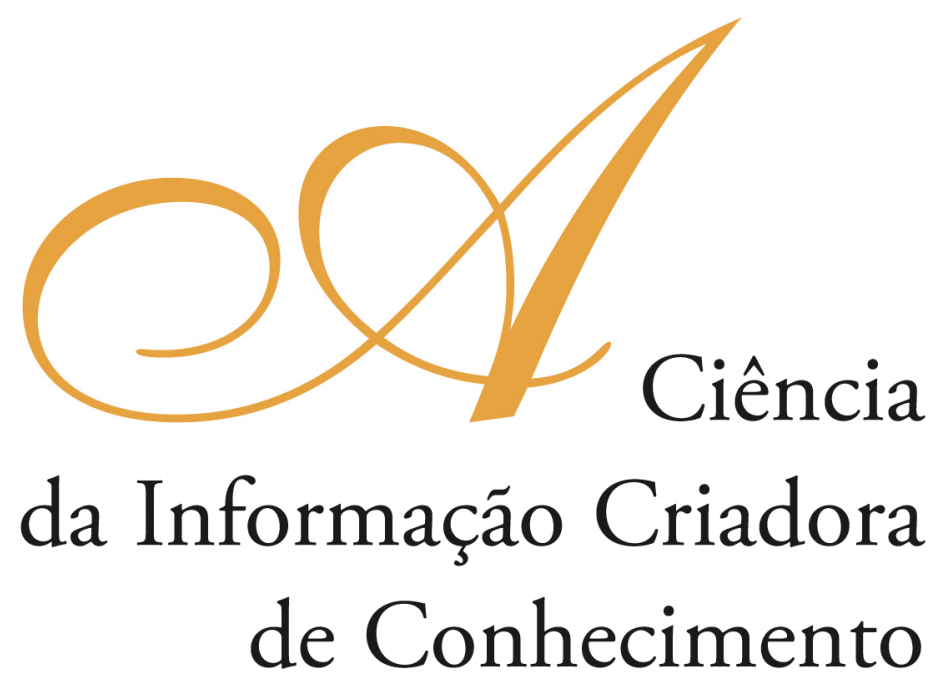

Vol. I

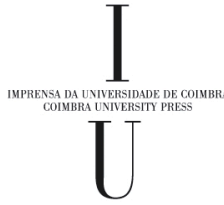

- COIMBRA 2009 


\title{
LA GESTIÓN DE LA CALIDAD EN EL CURRICULUM DE LOS Títulos DE GRADo y MÁSTER EN Biblioteconomía y Documentación
}

\author{
Alicia Arias Coello \\ Universidad Complutense de Madrid (Espanha) \\ José Simón Martín \\ Universidad Complutense de Madrid (Espanha)
}

\section{Resumen}

En esta comunicación se presenta la importancia que tiene la disciplina de la gestión de la calidad en los estudios de grado y de máster en el área académica de la Información y Documentación. Para su realización se han analizado 19 programas de grado y 21 de Máster adaptados al Espacio Europeo de Educación Superior (EEES) y ofertados por universidades públicas y privadas de España, Portugal y el Reino Unido.

En el caso del Reino Unido, además, las titulaciones elegidas debían cumplir la condición de estar acreditadas por el Chartered Institute of Library and Information Professionals (CILIP).

Como conclusión podemos decir que en 7 de los programas de grado se ofrecen asignaturas específicas sobre gestión de la calidad y, en el resto, la calidad se estudia como un contenido específico dentro de asignaturas dedicadas a la gestión de unidades de información.

El resultado en obtenido en los programas de Máster, es que la materia de gestión de la calidad aparece de forma específica en el currículum en 5 de ellos.

En conclusión, el análisis de la literatura y de los casos concretos de las universidades estudiadas, nos indica que el diseño de las nuevas titulaciones de información y documentación adaptadas al EEES, se ha ampliado con disciplinas orientadas a las tareas propias del gestor, sin abandonar las materias técnicas clásicas. Esta ampliación responde a una carencia explícita en una profesión (bibliotecario, archivero, documentalista) en la que existen buenos técnicos, pero pocos gestores con plenos conocimientos en temas específicos como es la gestión de la calidad, el marketing o la planificación y sus herramientas.

\begin{abstract}
This paper demonstrates the important role that quality management plays in Bachelor's and Master's degree studies in Library and Information Science (LIS). 19 Bachelor's and 21 Master's degree programs offered by public and private universities in Spain, Portugal and the United Kingdom were analyzed. All selected programs complied with the requirement of having been adapted to the European Space for Higher Education (ESHE). In addition, in the case of the United Kingdom, they had to be accredited by the Chartered Institute of Library and Information Professionals (CILIP). The results show that in 7 of the Bachelor's programs offered in the countries under study, there is a specific subject relating to quality management and, in the rest, quality is studied as a specific part of information unit management. In the case of Master's programs, the subject of quality management is specifically offered in 5 curricula out of the total number of programs analyzed.
\end{abstract}


To conclude, analysis of the literature and the specific cases of the universities studied shows that, in the design of the new information science degrees adapted to the ESHE, the study of management tasks has increased while maintaining the traditional subjects. This increase is a response to an explicit need in the profession (librarian, archivist, etc.) where good technical people can be found, but very few managers have extensive knowledge of specific subjects such as quality management, marketing or planning and the corresponding tools.

\section{Introducción}

En Europa la formación en el área de la Biblioteconomía y la Documentación se ha caracterizando por la diversidad de tradiciones, enfoques, estructura de los programas, niveles formativos, contenidos curriculares, duración de los estudios, o modelos de enseñanza aplicados (Audunson 2005, Kajberg 2007 ); sin embargo, el establecimiento de un Espacio Europeo de Educación Superior (EEES), puede llegar a ser una oportunidad para reducir esta diversidad unificando enfoques, tanto en la formación como en las competencias que debe reunir el profesional de la información. Esta armonización tendría la ventaja de favorecer la movilidad de estudiantes y profesionales dentro de Europa.

En este sentido fueron muy importantes las dos iniciativas que se llevaron a cabo en Europa. La primera de ellas fue el European LIS Curriculum Project, realizado en 2005 conjuntamente por la Royal School of Library and Information Science de Copenhague y la European Association for Library and Information Education and Research (EUCLID). La segunda iniciativa fue realizada con el patrocinio del European Council of Information Associations (ECIA) que elaboró el Euroreferencial en Información y Documentación, documento que es revisado periódicamente por la French Association of Library and Information Professionals (ADBS).

El Euroreferencial identifica, desde una perspectiva europea, las competencias y aptitudes que deberían poseer las personas que deseen ejercer su profesión en el área de la información y la documentación. Este documento tiene un carácter fundamental para la elaboración de los planes de estudio adaptados al EEES y para la definición del perfil del egresado, el cual debe responder a los resultados establecidos previamente, desde la concepción de las competencias y habilidades. Estos resultados se basan tanto en el conocimiento y en la comprensión de los distintos aspectos de la profesión, como en el desarrollo de aptitudes que le posibiliten el desempeño de distintas actividades laborales en el ámbito de la profesión para la que se ha formado a lo largo de sus estudios.

Tanto el Euroreferencial como el European LIS Curriculum Project van a permitir avanzar en la nueva concepción del profesional de la información que, de acuerdo con Abels et al. (2003), es un profesional que utiliza de manera estratégica la información en su trabajo para avanzar en la misión de la organización; para ello, desarrolla, despliega y gestiona los recursos y servicios de información y emplea la tecnología como una herramienta crítica para conseguir sus objetivos.

El Euroreferencial establece cinco grupos de competencias de aprendizaje. Dentro del grupo de gestión, y en el apartado de "diagnóstico y evaluación", establece las competencias adecuadas para asegurar que los futuros profesionales de la información y documentación sean capaces de "Identificar los puntos fuertes y los puntos débiles 
de un producto o de un servicio, de un sistema documental, de una organización; elaborar, establecer y utilizar indicadores de evaluación; gestionar el cuadro de mando de un servicio, asumir una gestión de la calidad de los productos, servicios y sistemas documentales de una organización”. Es importante hacer notar que los contenidos de este apartado sobre gestión de la calidad aparecen como competencias específicas en los cuatro niveles de aprendizaje, que incluyen el Grado, el Posgrado, el Doctorado y la Formación Continuada.

Como señalan Georgy et al. (2005) en el European LIS Curriculum Project, la materia de gestión de la calidad constituye un elemento esencial en el curriculum de las titulaciones de información y documentación, sobre todo en la de máster, ya que el control y el aseguramiento de la calidad deben ser considerados como elementos estratégicos en las bibliotecas pues su conocimiento, por parte de los estudiantes, les proporciona una comprensión global de la calidad aplicada a los servicios que debe incluir la parte teórica y la práctica con el fin de que aprendan a utilizar las herramientas para el aseguramiento de la calidad, sabiendo que las dimensiones de dichos servicios son el acceso, la comunicación, la competencia y la cortesía del personal, la credibilidad, la fiabilidad, la responsabilidad, la seguridad, los elementos tangibles y el conocimiento de la satisfacción de los usuarios.

Partiendo de las anteriores premisas, queremos presentar en esta comunicación el caso concreto de la introducción de la disciplina de gestión de la calidad en los estudios conducentes a la obtención del título de grado y en los estudios de máster en el área de la Información y la Documentación, tomando como referencia la presencia de estos estudios en España, Portugal y el Reino Unido. La elección de estos tres países se ha determinado por el hecho de que en otros países europeos la presencia de la disciplina, o de sus contenidos, no está presente de manera tan regular como en los países citados, hecho que puede favorecer la distorsión de la parte del estudio comparativo, ya que para cada uno de los países propuestos se comparan los contenidos de dicha disciplina y su adecuación con el entorno de aplicación de los conceptos, y de las herramientas de la gestión de la calidad en las distintas unidades de información.

En la introducción de la gestión de la calidad en una titulación, debemos distinguir dos situaciones. En primer lugar, el concepto de calidad como el grado en el que las características inherentes de un proceso, de un servicio, de una organización, de un producto, etc. cumple con unos requisitos esperados. Esta filosofía debería ser un enfoque que tendría que permear de forma transversal a todas las demás disciplinas curriculares; como por ejemplo en la catalogación, gestión de la información, etc.

En segundo lugar, el estudio de la calidad o de la gestión de la calidad como disciplina independiente en el currículo, debería incluir todos aquellos enfoques, conceptos, y metodologías que posteriormente van a ser útiles a los titulados como profesionales de la documentación e información en el entorno de su trabajo.

\section{Metodología}

Para la realización de este estudio se seleccionaron las titulaciones de grado y de máster que se imparten, actualmente, en las universidades públicas y privadas de Espańa, Portugal y Reino Unido. Los criterios de selección aplicados fueron: 
- Que la titulación ofertada por la universidad estuviera adaptada al EEES.

- Que la titulación dispusiera de una mínima información disponible en internet: objetivos de la enseñanza, plan de estudios, y contenido de las asignaturas.

- Que los objetivos y el contenido de la titulación pudieran ser incluidos en el área de la biblioteconomía, archivos o documentación.

En una primera etapa se identificaron los programas de grado y máster de cada uno de los tres países, para ello, y en el caso de España, se utilizó la relación de universidades que se recogen en el Libro Blanco sobre el Título de Grado en Información y Documentación (2004) y, para el caso del Reino Unido, se analizaron aquellas titulaciones que están acreditadas por el Chartered Institute of Library and Information Professionals (CILIP); por último, en el caso de Portugal se identificaron todas las universidades a las que se puede acceder a través de internet. Después de visitar los sitios web de cada uno de los programas, se identificaron diecinueve titulaciones de grado y veintiuna de máster que cumplen con los criterios de selección indicados anteriormente. En el apéndice 1 se muestra una lista de cada uno de estos programas y su dirección URL.

En una segunda etapa, para cada programa seleccionado se recogieron los objetivos generales, el plan de estudios, y toda la información disponible sobre cada asignatura, materia y/o modulo docente. Para analizar la presencia de la disciplina de calidad dentro del plan de estudio se llevaron a cabo dos revisiones; en la primera se comprobó si existían asignaturas o materias específicas en las que apareciera la palabra calidad, o las expresiones gestión de la calidad, o evaluación de la calidad. En aquellos planes de estudio en los que no existen asignaturas específicas, se analizó el contenido de todas las asignaturas del "curriculum" con la finalidad de encontrar, en el contenido de alguna de ellas, aspectos o referencias a alguno de los siguientes términos relacionados con la gestión de la calidad: calidad, gestión de la calidad, herramientas para el control de la calidad, autoevaluación de los servicios frente a un modelo o referencial de la calidad, estándares de calidad y certificación (Normas Internacionales ISO de la serie 9000), calidad en los servicios de las unidades de información y satisfacción de usuarios y servicios bibliotecarios.

\section{Resultados}

La adaptación de los estudios universitarios al EEES determina que los países participantes se comprometan, entre otros, a implantar un sistema de créditos comunes (el European Credit Transfer System ECTS), y la estructuración de los estudios de enseñanza superior en tres ciclos, uno de Grado con una duración mínima de tres años o 180 créditos ECTS, un segundo ciclo de Máster y un tercer ciclo de Doctorado.

De los 19 programas de formación de primer ciclo (Licenciatura, Grado o Bachelor) seleccionados, se encontraron siete asignaturas con la denominación de calidad. En la Tabla 1 se describe la Universidad a la que pertenece el programa, así como su posición en el curriculum y la duración en ECTS. 
Tabla 1 - Asignaturas pertenecientes a titulaciones de grado en cuya denominación aparece la palabra calidad

\begin{tabular}{|l|l|c|c|}
\hline \multicolumn{1}{|c|}{ Universidad } & \multicolumn{1}{c|}{ Asignatura/ materia } & Curso & ECTS \\
\hline Universidad Alcalá de Henares & Marketing y calidad de servicio & $1^{\circ}$ & 6 \\
\hline Universidad da Coruña & $\begin{array}{l}\text { Gestión de la calidad en organizaciones } \\
\text { documentales }\end{array}$ & $3^{\text {o }}$ & 6 \\
\hline Universitat de Barcelona & Avaluació i qualitat & $3^{\circ}$ & 9 \\
\hline Universidade Catolica de Portugal & Gestão da qualidade & & \\
\hline Universidade Portucalense & Normalizaçáo e gestão da qualidade & $2^{\circ}$ & 4 \\
\hline Universidade Fernando Pessoa & Normalização e gestão da qualidade & $1^{\circ}$ & 5 \\
\hline Universidade de Aveiro & Gestáo da qualidade. & $2^{\circ}$ & 6 \\
\hline
\end{tabular}

Los objetivos que se plantean en estas asignaturas de gestión de la calidad suelen ser, entre otros, que los estudiantes conozcan y aprendan las diferentes perspectivas de la calidad, así como los sistemas de la calidad que se aplican a las unidades de información, la autoevaluación y la certificación de las unidades de información, y la importancia de los estudios de satisfacción de los usuarios junto con los métodos que se utilizan.

En el resto de los doce programas analizados, la disciplina de gestión de la calidad aparece en el contenido de asignaturas que están relacionadas con la gestión, dirección, o evaluación de unidades de información, siendo su amplitud muy variable.

En el caso de los programas de postgrado se analizaron veintiuna titulaciones de Máster, apareciendo asignaturas o materias con la palabra calidad en cinco de ellos. En la Tabla 2 se presenta el nombre de las materias o asignaturas, el nombre del programa, la universidad en donde se imparte y el número de créditos ECTS que tiene asignada.

Tabla 2 - Asignaturas pertenecientes a estudios de Máster en cuya denominación aparece la palabra calidad

\begin{tabular}{|l|l|l|c|}
\hline \multicolumn{1}{|c|}{ Universidad } & \multicolumn{1}{|c|}{ Nombre del Programa } & \multicolumn{1}{c|}{ Asignatura/ materia } & ECTS \\
\hline Universidad Carlos III & $\begin{array}{l}\text { Máster en Bibliotecas y } \\
\text { Servicios de Información } \\
\text { Digital }\end{array}$ & $\begin{array}{l}\text { Sistemas de gestión de la } \\
\text { calidad }\end{array}$ & 3 \\
\hline Universidad de Granada & $\begin{array}{l}\text { Máster en Información } \\
\text { Científica: tratamiento, } \\
\text { acceso y evaluación }\end{array}$ & $\begin{array}{l}\text { Evaluación de la calidad y } \\
\text { certificación de bibliotecas y } \\
\text { servicios de información }\end{array}$ & 3 \\
\hline Universidad Zaragoza & $\begin{array}{l}\text { Máster en Gestión de } \\
\text { Unidades y Servicios } \\
\text { de Información y de } \\
\text { Documentación }\end{array}$ & $\begin{array}{l}\text { Evaluación de unidades y } \\
\text { servicios de información }\end{array}$ & 4,5 \\
\hline $\begin{array}{l}\text { Universidad Complutense } \\
\text { de Madrid }\end{array}$ & $\begin{array}{l}\text { Máster en Gestión de la } \\
\text { Documentación, Bibliotecas } \\
\text { y Archivos }\end{array}$ & $\begin{array}{l}\text { Gestión de la calidad y } \\
\text { satisfacción de usuarios. }\end{array}$ & 4,5 \\
\hline $\begin{array}{l}\text { Universidad Portucalense } \\
\text { Infante Don Henrique }\end{array}$ & $\begin{array}{l}\text { Mestrado em Ciências } \\
\text { da Informação e da } \\
\text { Documentaçáo }\end{array}$ & $\begin{array}{l}\text { Inovaçáo e gestão da } \\
\text { qualidade }\end{array}$ & 7,5 \\
\hline
\end{tabular}


Al igual que en el caso de los estudios de grado, la disciplina de gestión de la calidad puede aparecer como contenido en asignatura relacionadas con la gestión o el marketing de las unidades de información.

\section{Discusión y conclusiones}

Del análisis llevado a cabo sobre las titulaciones de primer y segundo ciclo del área de información y documentación, resulta llamativo el hecho de que una parte importante de los planes de estudios dediquen su atención a disciplinas relacionadas con aspectos referidos a la gestión de unidades de información: gestión, gestión del conocimiento, planificación, marketing, evaluación, calidad, organización, estudios de usuarios, etc., lo cual estaría de acuerdo con los planteamientos de la IFLA y otras organizaciones como la Special Library Association (SLA).

El intento actual de creación de un Espacio Europeo de Educación Superior, y sobre todo el hecho de que las titulaciones de primer ciclo (Grado, Licenciado, o Bachelor) y de segundo ciclo (Máster) hayan de estar orientadas a la adquisición de calificaciones profesionales significativas para el mercado de trabajo, nos brinda una oportunidad inmejorable para reducir la variabilidad en los enfoques y en los contenidos de los programas de enseñanza en Europa; sin embargo, como se desprende del análisis aquí realizado sobre la introducción de la disciplina de la gestión de la calidad en los "curricula" de información y documentación, todavía existe una gran variabilidad en lo que se refiere al ciclo de enseńanza y al contenido de esta asignatura.

Así mismo, y de acuerdo con lo que se indica en el Euroreferencial, los contenidos de esta materia deberían estar presentes en todos los ciclos de formación, siguiendo los niveles de competencia de aprendizaje indicados en dicho documento; ya que, como demuestra la literatura que existe sobre la implantación en la práctica de sistemas de gestión de calidad y el empleo de sus herramientas en unidades de información, muchos gestores de bibliotecas y archivos han demostrado un interés especial por estos temas y un gran empeño en la implantación de sistemas de gestión de la calidad en sus unidades de información, con el fin de conseguir una mejora continua en el servicio desempeñado.

De los resultados obtenidos y de los datos obtenidos de la literatura utilizada, se presentan las siguientes conclusiones:

El análisis de la literatura y de los casos concretos de las universidades estudiadas, nos indica que el diseño de las nuevas titulaciones de información y documentación adaptadas al EEES han ampliado las disciplinas orientadas a las tareas propias del gestor, sin abandonar las doctrinas técnicas que dan sentido a la profesión.

En este estudio se ha constatado que los contenidos sobre la gestión de la calidad se encuentran presentes en el grado y en el posgrado de la mayoría de los contenidos de esta disciplina en el ámbito de los tres países analizados, formando parte, en la mayoría de los casos, de un módulo mayor en el que se incluyen las disciplinas de planificación, gestión, planificación o márketing.

Entre los contenidos que aparecen con mayor frecuencia dentro de la gestión de la calidad son la aplicación de la Norma Internacional ISO 9001 en las unidades de 
información, y la medida del grado de satisfacción de los usuarios con los servicios y productos ofrecidos por estas unidades.

\section{Referências bibliográficas}

Abels, E., Jones, R., Latham, J., Magnoni, D., Marshall, J.G. (2003). "Competencies for Information Professionals of the 21 st Century”. Disponible en: http://www.sla.org/PDFs/ Competencies2003_revised.pdf

Audunson R. (2007). LIS and the Creation of a European Educational Space. Journal of Librarianship and Information Science. 2005; 37 (4); 171-174.

European Curriculum Reflections on Library and Information Science Education. Edited by Leif Kajberg and Leif Lørring. (2005). The Royal School of Library and Information Science. Copenhagen

Euroguide LIS: Competencies and aptitudes for European information professionals, by the European Council of Information Associations (ECIA). Vol.1, Guide n ${ }^{\circ}$ 8, (2004). Disponible en http://www.adbs.fr/l-euroreferentiel-en-cinq-groupes-de-competences-27624.htm?RH= METIER8REFCOMP (consultada el 20-05-09)

Georgy U, Lepik A, Petuchovait R. (2005) Library Management in European Curriculum. Reflections on Library and Information Science Education. Ed. by Leif Kajberg and Leif Lørring. Copenhagen: The Royal School of Library and Information Science.

Kajberg L. (2007) The European LIS Curriculum Project: an overview. Journal of Education for Library and Information Science. (spring) 48 (2); 68-81.

Guidelines for Professional Library/Information Educational Programs. Third revised draft, 2003 with subsequent minor revisions. These guidelines were approved by IFLA's Professional Board in December 2000. Since then minor updates have been made by the IFLA Section on Education and Training. These guidelines are in effect a revision of the «Standards for Library Schools» published by IFLA in 1976 for the Section on Education and Training. Disponible en: http://archive.ifla.org/VII/s23/bulletin/guidelines.htm\#curriculum (consultada el 21-06-09)

Título de grado en Información y Documentación. Madrid: Agencia Nacional de Evaluación de la Calidad y Acreditación, 2004. Disponible en: http://www.aneca.es/modal_eval/docs/ conver_biblio.pdf. 
Apéndice: Listado de programas seleccionados para este estudio

Tabla 3: PORTUGAL

\begin{tabular}{|c|c|c|}
\hline UNIVERSIDAD & PROGRAMA GRADO & PROGRAMA MÁSTER \\
\hline $\begin{array}{l}\text { Universidade de } \\
\text { Coimbra }\end{array}$ & $\begin{array}{l}\text { Ciências da Informaçäo } \\
\text { Arquivistica e } \\
\text { Biblioteconómica } \\
\text { https://woc.uc.pt/fluc/ } \\
\text { course/planocurricular. } \\
\text { do?courseId }=218 \\
\end{array}$ & \\
\hline Universidade do Porto & $\begin{array}{l}\text { Licenciatura em Ciência da } \\
\text { Informaçäo } \\
\text { http://sigarra.up.pt/flup/ } \\
\text { cursos_geral.FormView?P_ } \\
\text { CUR_SIGLA=LCINF }\end{array}$ & $\begin{array}{l}\text { Mestrado em Ciências da } \\
\text { Informaçäo } \\
\text { http://sigarra.up.pt/flup/cursos_geral. } \\
\text { FormView?P_CUR_SIGLA=MCI }\end{array}$ \\
\hline Universidade de Évora & $\begin{array}{l}\text { Licenciatura em Ciências da } \\
\text { Informaçáo e Documentaçáo } \\
\text { http://www.ip.uevora. } \\
\text { pt/es/oferta_formativa/11_- } \\
\text { ciclo/detalhe_do_curso/ } \\
\text { (codigo)/148 }\end{array}$ & $\begin{array}{l}\text { Mestrado em Ciências da } \\
\text { Informaçäo e da Documentaçäo } \\
\text { http://www.sac.uevora.pt/sac/estudos_ } \\
\text { pos_graduados/mestrados/cursos/ } \\
\text { cursos_de_edicoes_2009_2010/ } \\
\text { cursos/curso/(id)/289/(ano_lectivo)/ } \\
\text { 2009/(tipo)/bolonha }\end{array}$ \\
\hline $\begin{array}{l}\text { Universidade Católica } \\
\text { Portuguesa }\end{array}$ & $\begin{array}{l}\text { Licenciatura em Ciência da } \\
\text { Informação e Documentaçäo } \\
\text { http://www.facfil.ucp. } \\
\text { pt/1 ciclo/cid/ }\end{array}$ & $\begin{array}{l}\text { Mestrado em Ciências da } \\
\text { Informaçấo e da Documentaçáo } \\
\text { http://www.braga.ucp.pt/site/custom/ } \\
\text { template/ucptplfac.asp?SSPAGEID=1 } \\
\text { 321\&lang=1 \&artigoID }=1564\end{array}$ \\
\hline $\begin{array}{l}\text { Universidade } \\
\text { Portucalense Infante } \\
\text { Don Henrique }\end{array}$ & $\begin{array}{l}\text { Licenciatura em Ciência da } \\
\text { Informaçäo } \\
\text { http://wwwa.uportu.pt/ } \\
\text { siaa/Licenciaturas/ciencia_ } \\
\text { informacao.pdf }\end{array}$ & $\begin{array}{l}\text { Mestrado em Ciências da } \\
\text { Informação e da Documentaçäo } \\
\text { http://wwwa.uportu.pt/ } \\
\text { siaa/Mestrados/Informacao_ } \\
\text { Documentacao.pdf }\end{array}$ \\
\hline $\begin{array}{l}\text { Universidade Fernando } \\
\text { Pessoa }\end{array}$ & $\begin{array}{l}\text { Licenciatura em Ciência da } \\
\text { Informaçáo e Documentação } \\
\text { http://www.ufp.pt/index. } \\
\text { php?option=com_content } \& \\
\text { view=article\&id }=138 \% 3 \mathrm{Aci} \\
\text { encias-da-informacao-e-da- } \\
\text { documentacao\&catid }=37 \% 3 \mathrm{~A} \\
\text { licenciaturas } \& \text { Itemid }=69\end{array}$ & $\begin{array}{l}\text { Mestrado em Ciências da } \\
\text { Informaaçáo e da Documentaçäo } \\
\text { http://www.ufp.pt/index. } \\
\text { php?option=com_content\&view=ar } \\
\text { ticle\&id }=815 \% 3 \text { A2o-ciclomestrado- } \\
\text { em-ciencias-da-informacao-e-da-docu } \\
\text { emntacao\&catid=86\%3Amestrados\& } \\
\text { Itemid=79 }\end{array}$ \\
\hline Universidade de Aveiro. & $\begin{array}{l}\text { Licenciatura em } \\
\text { Documentaçáa } \boldsymbol{e} \\
\text { Arquivistica } \\
\text { http://www.ua.pt/ } \\
\text { guiaonline/PageCourse. } \\
\text { aspx?id=61\&b=1\&lg=pt }\end{array}$ & \\
\hline $\begin{array}{l}\text { Universidade Nova de } \\
\text { Lisboa }\end{array}$ & & $\begin{array}{l}\text { Mestrado em Ciências da } \\
\text { Informaçấo e da Documentaçäo } \\
\text { http://www.unl.pt/guia/2008/fcsh/c- } \\
4102\end{array}$ \\
\hline
\end{tabular}




\section{Tabla 4 - ESPAÑA}

\begin{tabular}{|c|c|c|}
\hline UNIVERSIDAD & PROGRAMA GRADO & PROGRAMA MÁSTER \\
\hline $\begin{array}{l}\text { Universidad Alcalá } \\
\text { de Henares }\end{array}$ & $\begin{array}{l}\text { Grado en Información } y \\
\text { Documentación } \\
\text { http://www.uah.es/acceso_- } \\
\text { informacion_academica/primero_} \\
\text { segundo_ciclo/matricula_I_II_ciclo/ } \\
\text { planes.asp?cd=109\&plan=G640 }\end{array}$ & \\
\hline $\begin{array}{l}\text { Universidad da } \\
\text { Coruña }\end{array}$ & $\begin{array}{l}\text { Grado en Información } \boldsymbol{y} \\
\text { Documentación } \\
\text { http://hum } 236 . c d f . u d c . \\
\text { es/fh/021c3195e00051b01/ } \\
\text { 0524fa9b2a0a49e01/ } \\
\text { 0524fa9c510b89737/ } \\
\text { 0524fa9c510c45907.php } \\
\end{array}$ & \\
\hline $\begin{array}{l}\text { Universidad Carlos } \\
\text { III }\end{array}$ & $\begin{array}{l}\text { Grado en Información } y \\
\text { Documentación } \\
\text { http://www.uc } 3 \mathrm{~m} . \mathrm{es} / \text { portal/page/ } \\
\text { portal/titulaciones_grado/info_doc }\end{array}$ & $\begin{array}{l}\text { Máster en Bibliotecas y Servicios } \\
\text { de Información Digital } \\
\text { http://www.uc3m.es/portal/page/ } \\
\text { portal/postgrado_mast_doct/ } \\
\text { masters/Master_Bibliotecas_y_- } \\
\text { Servicios_Informacion_Digital }\end{array}$ \\
\hline $\begin{array}{l}\text { Universidad de } \\
\text { Salamanca }\end{array}$ & $\begin{array}{l}\text { Grado en Información } \boldsymbol{y} \\
\text { Documentación } \\
\text { http://www.usal.es/webusal/node/474 }\end{array}$ & \\
\hline $\begin{array}{l}\text { Universitat de } \\
\text { Barcelona }\end{array}$ & $\begin{array}{l}\text { Grau en Informació y Documentació } \\
\text { http://www.ub.edu/graus/index. } \\
\text { php?option }=\text { com_content } \& \text { task=view } \\
\text { \&id=57\&Itemid }=0\end{array}$ & \\
\hline $\begin{array}{l}\text { Universidad de } \\
\text { Zaragoza }\end{array}$ & $\begin{array}{l}\text { Grado en Información } \boldsymbol{y} \\
\text { Documentación } \\
\text { http://ebro3.unizar.es: } 8080 / \mathrm{acad} / \\
\text { FMPro?-DB=w_titulaciones. } \\
\text { fp } 5 \& \text {-lay=cgi\&-format }=\text { grado. } \\
\text { htm\&-error }=\text { error } 2 . \text { htm } \& \text { id } \\
\text { titulacion }=104,5 \&-\mathrm{Max}=25 \&- \\
\text { SortField=Orden } \& \text {-Find }\end{array}$ & $\begin{array}{l}\text { Máster en Gestión de Unidades } \\
\text { y Servicios de Información } y \text { de } \\
\text { Documentación } \\
\text { http://ebro3.unizar.es: } 8080 / \mathrm{acad} / \\
\text { FMPro?-DB=w_titulaciones. } \\
\text { fp } 5 \&-\mathrm{lay}=\mathrm{cgi} \& \text {-format }=\text { grado. } \\
\text { htm } \& \text {-error }=\text { error } 2 . \mathrm{htm} \& i d- \\
\text { titulacion }=249,5 \&-\mathrm{Max}=25 \& \text { - } \\
\text { SortField=Orden } \& \text {-Find }\end{array}$ \\
\hline $\begin{array}{l}\text { Universidad } \\
\text { Complutense de } \\
\text { Madrid }\end{array}$ & $\begin{array}{l}\text { Grado en Información } y \\
\text { Documentación } \\
\text { http://www.ucm.es/centros/webs/ } \\
\text { ebiblio/index.php?tp=T\%EDtulo } \% 20 \\
\text { de } \% 20 \mathrm{Grado} \% 20 \mathrm{en} \% 20 \text { Informaci } \% \mathrm{~F} \\
\text { 3n } \% 20 \mathrm{y} \% 20 \text { Documentaci } \% \mathrm{~F} 3 \mathrm{n} \& \mathrm{a}=\mathrm{d} \\
\text { ocencia\&d=18087.php }\end{array}$ & $\begin{array}{l}\text { Máster en Gestión de la } \\
\text { documentación, Bibliotecas } y \\
\text { Archivos } \\
\text { http://www.ucm.es/centros/webs/ } \\
\text { ebiblio/index.php?tp=Docencia\&a } \\
=\text { docencia\&d=2977.php }\end{array}$ \\
\hline $\begin{array}{l}\text { Universitat Oberta } \\
\text { de Catalunya }\end{array}$ & $\begin{array}{l}\text { Grau en Informació y Documentació } \\
\text { http://www.uoc.edu/estudis/graus/ } \\
\text { infodocu/presentacio/index.html }\end{array}$ & \\
\hline $\begin{array}{l}\text { Universitat de } \\
\text { València }\end{array}$ & $\begin{array}{l}\text { Grau en Informació y Documentació } \\
\text { http://www.uv.es/acces/doc/graus/ } \\
\text { Graus_Info_Documentacio.pdf }\end{array}$ & \\
\hline $\begin{array}{l}\text { Universidad de } \\
\text { Granada }\end{array}$ & & $\begin{array}{l}\text { Máster en Información Cientifica: } \\
\text { tratamiento, acceso y evaluación } \\
\text { http://oficinavirtual.ugr.es/apli/ } \\
\text { posgrado/detalle_ofic.jsp?ano }=200 \\
9 \& \mathrm{dto}=910 \& \text { plan }=56 \& \mathrm{num}=1\end{array}$ \\
\hline
\end{tabular}


Tabla 5 - GRAN BRETAÑA

\begin{tabular}{|c|c|c|}
\hline UNIVERSIDAD & PROGRAMA GRADO & PROGRAMA MÁSTER \\
\hline Aberystwyth University & $\begin{array}{l}\text { Information and Library } \\
\text { Studies (Single Honours) } \\
\text { http://www.dis.aber. } \\
\text { ac.uk/en/courses/bsc_ } \\
\text { information_and_library_- } \\
\text { studies.asp }\end{array}$ & \\
\hline Manchester University & $\begin{array}{l}\text { BA (Hons) Librarianship } \\
\text { http://www.hlss.mmu. } \\
\text { ac.uk/infocomms/ } \\
\text { undergraduate/ }\end{array}$ & $\begin{array}{l}\text { MA Library and Information } \\
\text { Management } \\
\text { http://www.hlss.mmu.ac.uk/ } \\
\text { infocomms/postgraduate/ }\end{array}$ \\
\hline Northumbria University & $\begin{array}{l}\text { Librarianship (BSc (Hons) } \\
\text { http://www.northumbria. } \\
\text { ac.uk/?view=CourseDetail } \\
\text { \&code=DUDLIB1 }\end{array}$ & $\begin{array}{l}\text { Information and Library } \\
\text { Management (MA/MSc) } \\
\text { http://www.northumbria.ac.uk/ } \\
\text { ?view=CourseDetail\&code=DTFILM6 }\end{array}$ \\
\hline $\begin{array}{l}\text { The Robert Gordon } \\
\text { Univversity. Aberdeen } \\
\text { Business School }\end{array}$ & & $\begin{array}{l}\text { MSc Information and Library } \\
\text { Studies } \\
\text { http://www.rgu.ac.uk/abs/ } \\
\text { postgraduate/page.cfm?pge= } 5352\end{array}$ \\
\hline $\begin{array}{l}\text { University of the West of } \\
\text { England. Bristol Institute } \\
\text { of technology }\end{array}$ & & $\begin{array}{l}\text { MSc Information and Library } \\
\text { Management } \\
\text { http://courses.uwe.ac.uk/gp5112/ }\end{array}$ \\
\hline University of Strahclyde & & $\begin{array}{l}\text { MSc in Information Management } \\
\text { http://www.strath.ac.uk/cis/ } \\
\text { courses/mscpgdipinformationand } \\
\text { librarystudiespostgraduate/ } \\
\text { managementandmarketingofils/ }\end{array}$ \\
\hline Leeds University & & $\begin{array}{l}\text { PG Dip/MA/MSc Information } \\
\text { Studies } \\
\text { http://prospectus.leedsmet.ac.uk/ } \\
\text { main/detail.htm?p=58\&ban=INSTM }\end{array}$ \\
\hline City University London & & $\begin{array}{l}\text { MA/MSc Library Science } \\
\text { http://www.soi.city.ac.uk/pgcourses/ } \\
\text { lis/index.html }\end{array}$ \\
\hline Loughborough University & & $\begin{array}{l}\text { Information and Library } \\
\text { Management } \\
\text { http://lboro.ac.uk/departments/ls/ }\end{array}$ \\
\hline Sheffield University & & $\begin{array}{l}\text { MA in Librarianship } \\
\text { http://www.shef.ac.uk/is/ } \\
\text { prospectivepg/courses/lib/index.html }\end{array}$ \\
\hline University College London & & $\begin{array}{l}\text { MA/Postgraduate Diploma in } \\
\text { Library and Information Studies } \\
\text { http://www.ucl.ac.uk/prosp-students/ } \\
\text { gradprospectus/arts-and-humanities/ } \\
\text { lais/ }\end{array}$ \\
\hline $\begin{array}{l}\text { John Moores University } \\
\text { Liverpool }\end{array}$ & & $\begin{array}{l}\text { Information and Library } \\
\text { Management } \\
\text { MA/MSc/Postgraduate diploma } \\
\text { http://www.livjm.ac.uk/courses/ } \\
\text { postgraduate/59652.htm }\end{array}$ \\
\hline
\end{tabular}

